\title{
Lipid rafts in the bovine milk fat globule membrane revealed by the lateral segregation of phospholipids and heterogeneous distribution of glycoproteins
}

\author{
Christelle Lopez ${ }^{a}$, Marie-Noëlle Madec ${ }^{a}$, Rafael Jimenez-Flores ${ }^{b}$ \\ a INRA, AGROCAMPUS OUEST, UMR 1253 Science et Technologie du Lait et de l'Oeuf, 65 rue de Saint-Brieuc, F-35000 Rennes, France \\ ${ }^{\mathrm{b}}$ California Polytechnic State University, San Luis Obispo, CA 93401, USA
}

\begin{abstract}
A B S T R A C T
This study reveals the lateral organisation of the milk fat globule membrane (MFGM). Using confocal laser scanning microscopy (CLSM) and a lipid soluble molecule, an exogenous phospholipid and two lectins as fluorescent probes we located triacylglycerols in the core of fat globules and investigated the organisation of the polar lipids and glycoproteins of the MFGM, in situ in milk. Lipid rafts corresponding to the lateral segregation of sphingolipids in liquid-ordered phases surrounded by liquid-disordered domains composed by the glycerophospholipids were observed in the MFGM. These lipid rafts which correspond to rigid sphingolipid-rich domains have a circular shape at room temperature. CLSM experiments revealed that glycoproteins and glycolipids are heterogeneously distributed around fat globules and that they are not located in the lipid rafts. The characterisations performed by in depth thin sectioning of fat globules and in dynamic as a function of time revealed chemical and structural heterogeneities in the MFGM. Schematic 3D and 2D representations of the MFGM are proposed and discussed. The physiological and nutritional consequences of the lateral organisation of polar lipids and glycoproteins in the MFGM are discussed but remain to be elucidated.
\end{abstract}

Keywords:

Membrane structure, Lipid raft, Fluorescence microscopy, Milk fat globule, Sphingomyelin, Phospholipid

\section{Introduction}

As an essential food for mammal newborns, milk is a natural emulsion in which lipids are present in the form of small droplets called milk fat globules (mean diameter $\sim 4 \mu \mathrm{m}$ ). Milk fat globules are natural vehicles to bring the triacylglycerols ( $\sim 98 \%$ of total lipids; important source of energy) and biologically active molecules such as polar lipids and proteins for nutritional and health aspects (Spitsberg, 2005). Moreover, the components of the biological membrane surrounding fat globules, known as the milk fat globule membrane (MFGM), are involved with anti-infection or anti-adhesion, and hence with protection of the neonate from various viral and bacterial infections. The MFGM is also a barrier against lipolytic hydrolysis and contributes to the physical stability of fat globules in milk. It is clear that the functional and nutritional properties of the MFGM result from its chemical composition and the lateral organisation of its constituents. However, few studies have investigated the organisation of the MFGM in situ in milk.

The MFGM results from the mechanisms of secretion of fat globules (for further detail, see the review of Heid and Keenan (2005)). The biochemical composition of the MFGM is quite well-known and its potentialities as a nutraceutical have been reviewed (Spitsberg, 2005). The MFGM consists of a complex mixture of (glyco)proteins (20-60\%), glycerophospholipids (15-33\% of the MFGM), sphingolipids, glycolipids (i.e. cerebrosides and gangliosides), triacylglycerols, sterols ( $0.3 \%$ of milk lipids), enzymes and other minor components (Keenan \& Patton, 1995). The glycerophospholipids of the MFGM are phosphatidylcholine (PC; 35$36 \%$ ), phosphatidylethanolamine (PE; 27-30\%), phosphatidylinositol (PI; 5-11\%) and phosphatidylserine (PS; 3-4\%). The major sphingolipid of the MFGM is sphingomyelin (SM; 25\% of polar lipids) (Christie, 1995; Lopez et al., 2008). There are also glycosphingolipids in the MFGM, such as glucosyl-ceramide and lactosyl-ceramide with amounts in the range 3-6\% (Fong, Norris, \& MacGibbon, 2007; Rombaut, Camp, \& Dewettinck, 2005). Complex biological lipids such as glycerophospholipids and sphingolipids are quantitatively minor constituents of milk fat but are of great interest because they define the structural properties of the MFGM surrounding the triacylglycerol core of fat globules. The protein composition of the bovine MFGM has recently been reported (Fong et al., 2007; Mather, 2000; Reinhardt \& Lippolis, 2006). Many of the MFGM proteins are glycoproteins such as butyrophilin (20-40\% of the total MFGM protein), heavily-glycosylated mucin-like glycoproteins MUC1 and MUC15, PAS6/7, and the heavily-glycosylated CD36 (Keenan \& Mather, 2006). The gly- 
cans associated with the proteins contribute to the outer glycocalyx of the MFGM.

From a structural point of view, the concept of the organisation of the MFGM as a trilayer (thickness: $10-20 \mathrm{~nm}$ ) is well accepted (Heid \& Keenan, 2005). An electron dense material on the inner membrane face is composed of proteins and polar lipids from the endoplasmic reticulum and the cytoplasm of the lactating cell. The outer part of the MFGM corresponds to a biological membrane organised as a bilayer, derived from the apical plasma membrane of the mammary epithelial cells that surrounds fat globules when they are secreted. Thus, the most widely accepted model for the bilayer would be the fluid mosaic model (Singer \& Nicolson, 1972). This model suggests that the phospholipid bilayer serves as a backbone of the membrane, which exists in a fluid state. On the basis of their chemical properties (hydrophobicity, polarity), the main components (proteins, phospholipids, sphingolipids, glycolipids, glycoproteins, cholesterol), are expected to be asymmetrically arranged in the MFGM. Authors have reported schematic twodimensional representations of the organisation of the MFGM which have evolved over time (Danthine, Blecker, Paquot, Innocente, \& Deroanne, 2000; Dewettinck et al., 2008; Evers, 2004; Lopez, Briard-Bion, et al., 2008; Michalski, Michel, Sainmont, \& Briard, 2001). However, the structure of the MFGM still remains a poorly understood aspect of the milk fat globules. More particularly, information on the lateral organisation of the polar lipids and other constituents in the MFGM is scarce.

The precise location of the main constituents of the MFGM remains hypothetical due to the lack in the methodologies allowing its characterisation in situ in milk. Much of the knowledge about the structure and molecular organisation of the MFGM has been obtained using morphological or biochemical techniques applied to isolated membranes obtained using physical or chemical techniques as reviewed in Evers (2004). The characterisation of the organisation of the components requires the use of microscopy techniques, i.e. transmission and scanning electron microscopy, (immuno)fluorescence microscopy which can be associated with a labelling step. Immunochemical strategies using antibodies against defined proteins (i.e. butyrophilin, adipophilin, xanthine oxidoreductase) have been used (Franke et al., 1981; Robenek et al., 2006). Lectin-labelled gold granules have also been used for probing glycoproteins (Horisberger, Rosset, \& Vonlanthen, 1977). Using confocal laser scanning microscopy, Lopez, BriardBion, Beaucher, and Ollivon (2008) developed a protocol allowing the labelling of milk phospholipids in milk and dairy products such as cheese. More recently, Evers et al. (2008) used lipophilic probes and fluorescent lectin wheat germ agglutinin (WGA) to investigate the organisation of the MFGM in milks from various species.

The objective of this study is to increase the knowledge concerning the lateral organisation of the polar lipids and glycoproteins in the membrane surrounding milk fat globules. Confocal laser scanning microscopy and adequate fluorescent dyes were used to characterise, in situ in milk, the organisation of the MFGM.

\section{Materials and methods}

\subsection{Samples}

The bovine milks ( $41.9 \pm 1.1 \mathrm{~g}$ fat $/ \mathrm{L})$ used in this study were obtained from a local dairy plant (Triballat, Noyal sur Vilaine, France). They corresponded to raw full-fat milks.

\subsection{Particle size measurements}

The fat globule size distributions in the milks were measured by laser light scattering using a Mastersizer 2000 (Malvern Instru- ments, Malvern, UK), equipped with an He/Ne laser $(\lambda=633 \mathrm{~nm})$ and an electroluminescent diode $(\lambda=466 \mathrm{~nm})$. The refractive index of milk fat was taken to be 1.460 at $466 \mathrm{~nm}$ and 1.458 at $633 \mathrm{~nm}$. The samples of milk (about $0.2 \mathrm{ml}$ ) were diluted in $100 \mathrm{ml}$ of water directly in the measurement cell of the apparatus in order to reach $10 \%$ obscuration. The casein micelles were dissociated by adding $1 \mathrm{ml}$ of $35 \mathrm{mM}$ EDTA/NaOH, pH 7 buffer to the milks, in the apparatus. The size distribution of fat globules were characterised by the volume-weighted average diameter $d_{43}$ defined as $\sum n_{i} d_{i}^{4} / \sum n_{i} d_{i}^{3}$, where $n_{i}$ is the number of fat globules of diameter $d_{i}$. The specific surface area $S=6 \varphi / d_{32}$, where is the volume fraction of milk fat and $d_{32}$ is the volume/surface average diameter defined as $\sum n_{i} d_{i}^{3} / \sum n_{i} d_{i}^{2}$ was calculated by the software.

\subsection{Zeta potential}

The apparent zeta potential of milk fat globules was calculated from the measurement of their electrophoretic mobility determined using a Zetasizer 3000 HS (Malvern Instruments) equipped with palladium electrodes and an avalanche photodiode detector as previously detailed in Lopez, Camier, and Gassi (2007). Samples were prepared by suspending $50 \mu \mathrm{l}$ milk in $10 \mathrm{ml}$ milk ultrafiltrate, then $300 \mu$ of this preparation was suspended in $10 \mathrm{ml}$ milk ultrafiltrate and introduced in the capillary tube for measurement. The analyses were performed in triplicate for each milk.

\subsection{Microstructural analysis}

The microstructural analysis was performed using an inverted microscope NIKON Eclipse-TE2000-C1si (NIKON, Champigny sur Marne, France) allowing confocal laser scanning microscopy (CLSM) and optical microscopy using differential interferential contrast (DIC) also called Nomarsky. Confocal experiments were performed using an argon laser operating at $488 \mathrm{~nm}$ excitation wavelength (emission was detected between 500 and $530 \mathrm{~nm}$ ) a He-Ne laser operating at $543 \mathrm{~nm}$ wavelength excitation (emission was detected between 565 and $615 \mathrm{~nm}$ ). The observations were performed using a $\times 40$ (NA 1.33) and a $\times 100$ (NA 1.4) oil immersion objectives.

Neutral lipids such as triacylglycerols were stained with the lipid-soluble Nile Red fluorescent dye (9-diethylamino-5H-benzoalpha-phenoxazine-5-one; Sigma-Aldrich, St Louis, USA), prepared with a concentration of $42 \mu \mathrm{g} / \mathrm{ml}$ acetone. About $100 \mu \mathrm{l}$ of the Nile Red solution were added to $1 \mathrm{ml}$ of milk (excitation laser $=543 \mathrm{~nm}$ ). The fluorescent dye $\mathrm{N}$-(lissamine rhodamine B sulfonyl) di-oleoyl-phosphatidylethanolamine (Rh-PE; Avanti polar lipids Inc., Birmingham, England) was used to label the phospholipids. About $20 \mu \mathrm{l}$ of the commercial solution were added to $0.5 \mathrm{ml}$ of milk (excitation laser $=543 \mathrm{~nm}$ ). The lectins Concanavalin A Alexa fluor 488 (ConA; Invitrogen, Cergy Pontoise, France) and wheat germ agglutinin Alexa fluor 488 (WGA; Invitrogen, Cergy Pontoise, France) were used to localise the carbohydrate moities in the milk fat globule membrane. ConA was mixed with sodium carbonate $0.1 \mathrm{M} \mathrm{pH} 8.3$ (VWR Prolabo, Fontenay sous bois, France) to reach a concentration of $5 \mathrm{mg} / \mathrm{ml}$. About $50 \mu \mathrm{l}$ of the ConA solution were added to $1 \mathrm{ml}$ of milk (excitation laser $=488 \mathrm{~nm}$ ). A $1 \mathrm{mg} / \mathrm{ml}$ solution of WGA has been prepared by dissolving the powder in PBS buffer 0.2 M pH 7.4 (sigma P4417, Sigma-Aldrich, St. Louis, USA). All the solutions containing the fluorescent dyes were kept frozen until utilisation. Low melting point agarose (Sigma, St. Quentin Fallavier, France) was prepared at $5 \mathrm{~g} / \mathrm{L}$ and stored at $45^{\circ} \mathrm{C}$ until utilisation.

After the labelling step, the samples were kept at room temperature for at least $2 \mathrm{~h}$ before the microstructural analysis. Then, $5 \mu \mathrm{l}$ of the milk stained with the fluorescent dyes were deposited onto the glass, slowly mixed with $20 \mu$ of the agarose and observed on 
the microscope. The microstructural analyses were performed at room temperature (e.g. $19 \pm 1^{\circ} \mathrm{C}$ ).

Differential interferential contrast (DIC) microscopy was used to visualize fat globules (localisation in milk, shape, size). DIC images were sometimes superimposed to the emission fluorescence recorded in the CLSM images. The two-dimensional images had a resolution of $512 \times 512$ pixels and the pixel scale values were converted into micrometers using a scaling factor. In the multiple labelled samples, different colours were used to locate the fluorescent molecules.

\section{Results}

\subsection{Milk fat globules: size distribution and zeta potential}

The size distribution of milk fat globules, determined using laser light scattering, ranged from 0.06 to about $10 \mu \mathrm{m}$, with a mean volume-weighted diameter of $4.1 \pm 0.1 \mu \mathrm{m}$ (Fig. 1 ). The fat globule size distribution appeared as being polydispersed and multimodal. It was composed by three peaks corresponding to populations of particles: (i) the smallest particles ranged from 0.06 to $0.3 \mu \mathrm{m}$ with a maximum at $120 \mathrm{~nm}$, (ii) from 0.3 to $1 \mu \mathrm{m}$ with a maximum at $0.6 \mu \mathrm{m}$ and (iii) the main population of fat globules ranged from
1 to about $10 \mu \mathrm{m}$ with a maximum at about $4 \mu \mathrm{m}$. Milk fat globules were also characterised under the microscope using differential interferential contrast (DIC). Fig. 1A shows the size distribution and the spherical shape of milk fat globules. The specific surface area of milk fat globules calculated from the laser light scattering experiments was $3.8 \pm 0.1 \mathrm{~m}^{2} / \mathrm{g}$ fat. As the milks which have been characterised in this study contained $41.9 \pm 1.1 \mathrm{~g}$ fat $/ \mathrm{L}$, the specific surface area of fat globules was $159.1 \pm 1.6 \mathrm{~m}^{2} / \mathrm{L}$ milk. The zeta potential of milk fat globules was $-10.5 \pm 0.5 \mathrm{mV}$, which is in accordance with previous results obtained for milk fat globules (Lopez et al., 2007). Both the size distribution and zeta potential of fat globules confirmed that the experiments were conducted with raw milks, i.e. milks without any mechanical or heat treatment after milking.

\subsection{Triacylglycerols in the core of fat globules}

Triacylglycerol molecules, which represent about 98\% of milk lipids, were labelled with the lipid soluble fluorescent dye Nile Red and their localisation in milk was investigated using confocal laser scanning microscopy (CLSM). Fig. 1B shows the emission fluorescence of Nile Red organised as dispersed areas with a spherical shape. The overlay of the images taken by DIC (Fig. 1A) and CLSM
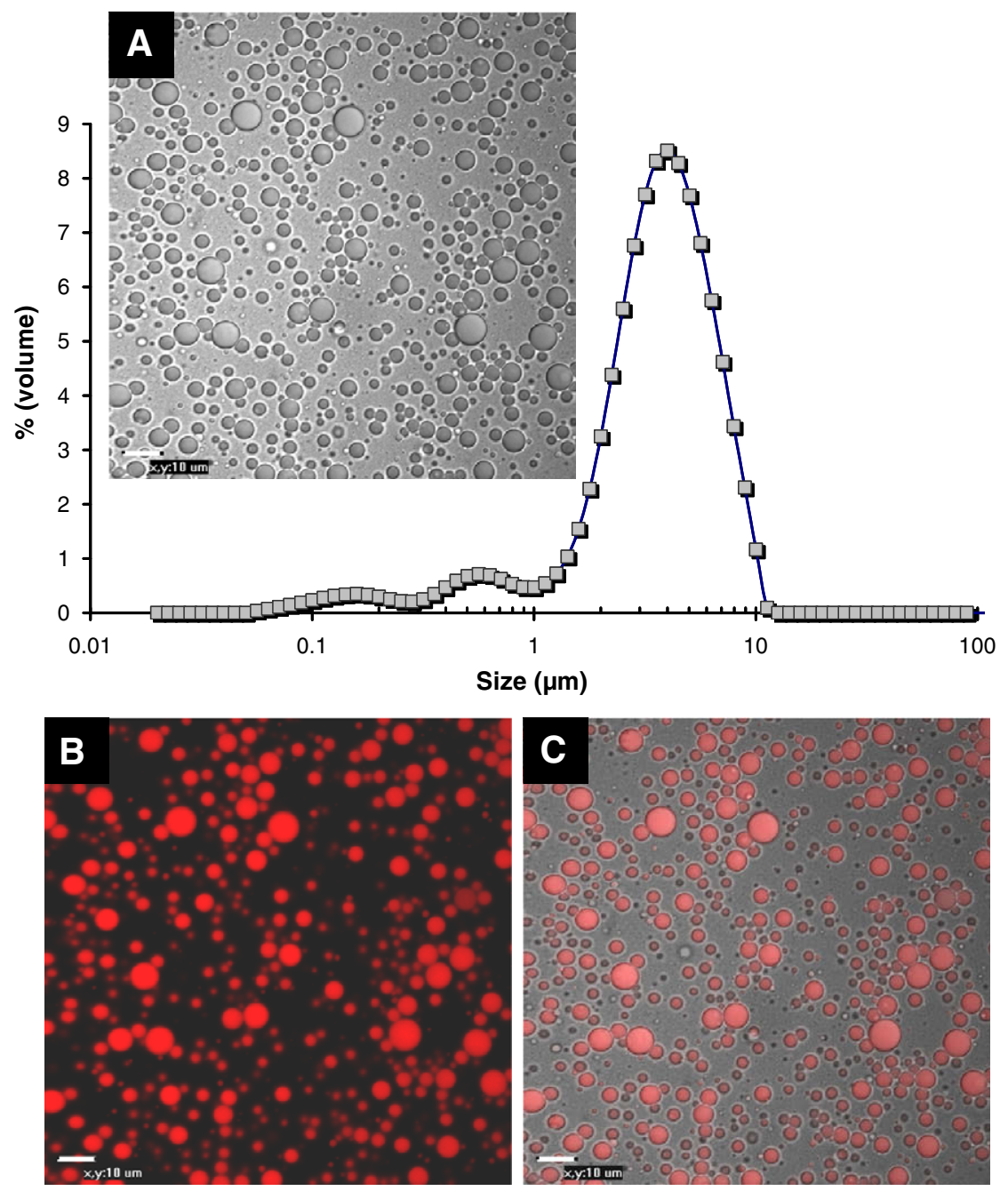

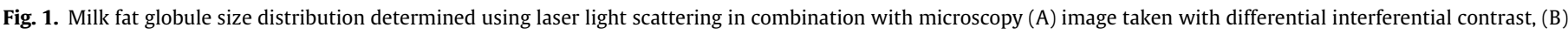

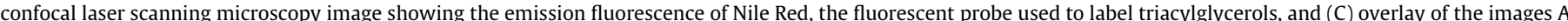

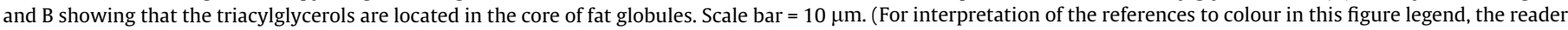
is referred to the web version of this article.) 
using Nile Red fluorescent dye confirmed that triacylglycerols are exclusively located in the core of fat globules (Fig. 1C). This is not surprising since triacylglycerols are hydrophobic molecules.

\subsection{Lateral organisation of polar lipids in the MFGM}

The lateral organisation of polar lipids which are located in the milk fat globule membrane (MFGM) surrounding fat globules was investigated using Rh-PE as fluorescent lipid probe. Fig. 2 shows the emission fluorescence of Rh-PE (Fig. 2A), the image of milk fat globules taken using DIC (Fig. 2B) and the overlay of the two images (Fig. 2C) showing that the emission fluorescence of $\mathrm{Rh}-\mathrm{PE}$ is located at the periphery of fat globules. Fig. 2 shows that the emission fluorescence of Rh-PE is not homogeneously distributed around fat globules. Some areas surrounding fat globules do not show the emission fluorescence of Rh-PE (Fig. 2). Since the exogenous dye Rh-PE is a phospholipid (PE) with a fluorescently labelled polar head group (rhodamine) which can incorporate in the bilayer of biological membranes, we deduced that the distribution of Rh-PE fluorescence intensity gave information on the localisation of milk phospholipids in the MFGM surrounding fat globules. The fluorescence of membrane-bound Rh-PE permitted the characterisation of different organisations of polar lipids around milk fat globules. In Fig. 3, fat globules noted A showed a red circle in two-dimensional representation, corresponding to a homogeneous distribution of the fluorescence dye in the MFGM surrounding fat globules. The interior of the sphere (in a 3D rep-
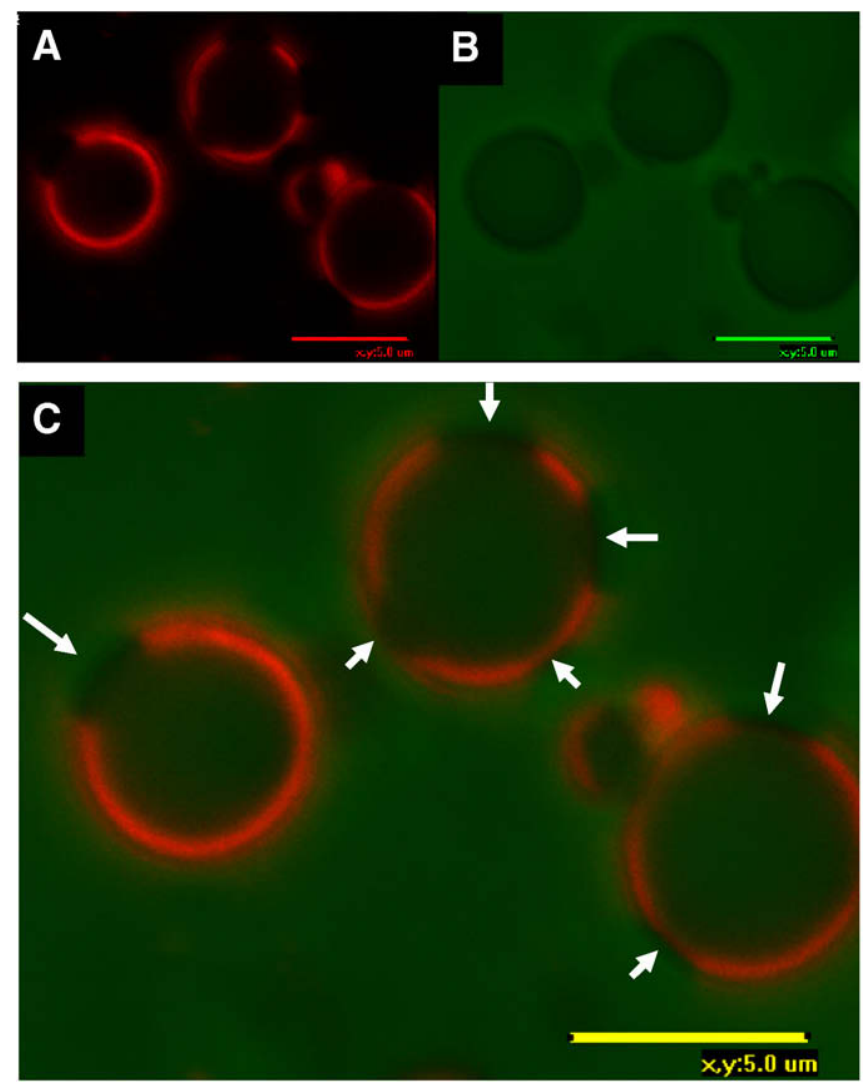

Fig. 2. Confocal laser scanning micrograph showing (A) the emission fluorescence of Rh-PE fluorescent dye detected using confocal laser scanning microscopy (red colour), (B) fat globules observed using differential interferential contrast (green colour), and (C) the overlay of images A and B showing the fluorescent dye integrated in the milk fat globule membrane surrounding fat globules, which are characterised in their equatorial plane. Scale bar $=5 \mu \mathrm{m}$. (For interpretation of the references to colour in this figure legend, the reader is referred to the web version of this article.)

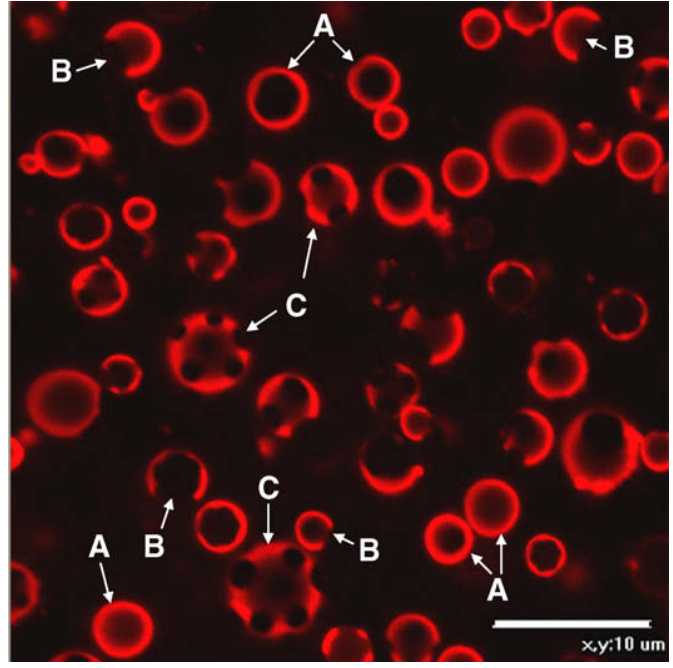

Fig. 3. Confocal laser scanning micrograph showing the emission fluorescence of Rh-PE fluorescent dye detected using confocal laser scanning microscopy (red colour). (A) Fat globules totally surrounded by Rh-PE emission fluorescence in their equatorial plane, (B) fat globules partially surrounded by Rh-PE emission fluorescence in their equatorial plane, and (C) fat globules characterised in their top or bottom in which non-fluorescent circular areas are observed in the MFGM. (For interpretation of the references to colour in this figure legend, the reader is referred to the web version of this article.)

resentation of fat globules), which is characterised in the equatorial plane and appears in black, corresponds to the triacylglycerols present in the core of fat globules (as shown in Fig. 1). In the case of the fat globules noted B the red circle was not full (Fig. 3). The black area observed around these fat globules characterised in their equatorial plane reveals that they were only partially surrounded by the fluorescent dye (Figs. 2 and 3). Fat globules noted $\mathrm{C}$ in Fig. 3 show a heterogeneous distribution of Rh-PE with the presence of black areas dispersed in the MFGM which are characterised by a circular shape. These fat globules are not characterised in their equatorial plane, but rather in their superior or inferior part which are covered by polar lipids. From 1 to 6 domains without Rh-PE emission fluorescence were observed at the surface of fat globules. Their diameter ranged from 0.6 to $2.7 \mu \mathrm{m}$, depending on the size of fat globules. Around milk fat globules of about $4 \mu \mathrm{m}$ diameter the domains ranged from 0.9 to $1.6 \mu \mathrm{m}$, with an average of $1.4 \pm 0.3 \mu \mathrm{m}$ (determined with image analysis). Domains with a larger diameter (2.5-2.7 $\mu \mathrm{m})$ were observed around fat globules of about $8 \mu \mathrm{m}$.

A three-dimensional (3D) characterisation of fat globules was performed by optical sectioning (i.e. depth imaging of fat globules) using both CLSM and DIC. Fig. 4 shows the emission fluorescence of Rh-PE (coloured in red) at different $z$-depths in the milk sample. This strategy allowed the characterisation of the organisation of phospholipids from the bottom (noted $z$ ) to the top (noted $z+4.5 \mu \mathrm{m}$ ) of a fat globule. The thickness of $4.5 \mu \mathrm{m}$ corresponds to the diameter of most of milk fat globules, according to Fig. 1. As already observed in Fig. 3, circular domains characterised by an absence of emission fluorescence of Rh-PE (i.e. non-coloured in red) appeared in the MFGM at both the bottom and the top of the fat globule. For intermediate $z$-depths the area surrounded by the emission fluorescence of Rh-PE corresponds to hydrophobic lipids, e.g. triacylglycerols, in the core of fat globules as already shown in Fig. 1C. Fig. 4 also shows the 3D characterisation of a small fat globule (diameter $\sim 3 \mu \mathrm{m}$ ). The bottom of the latter fat globule seems to be fully covered by Rh-PE while areas without emission fluorescence of Rh-PE were characterised at a higher $z$ levels, as indicated by an arrow in Fig. 4. 


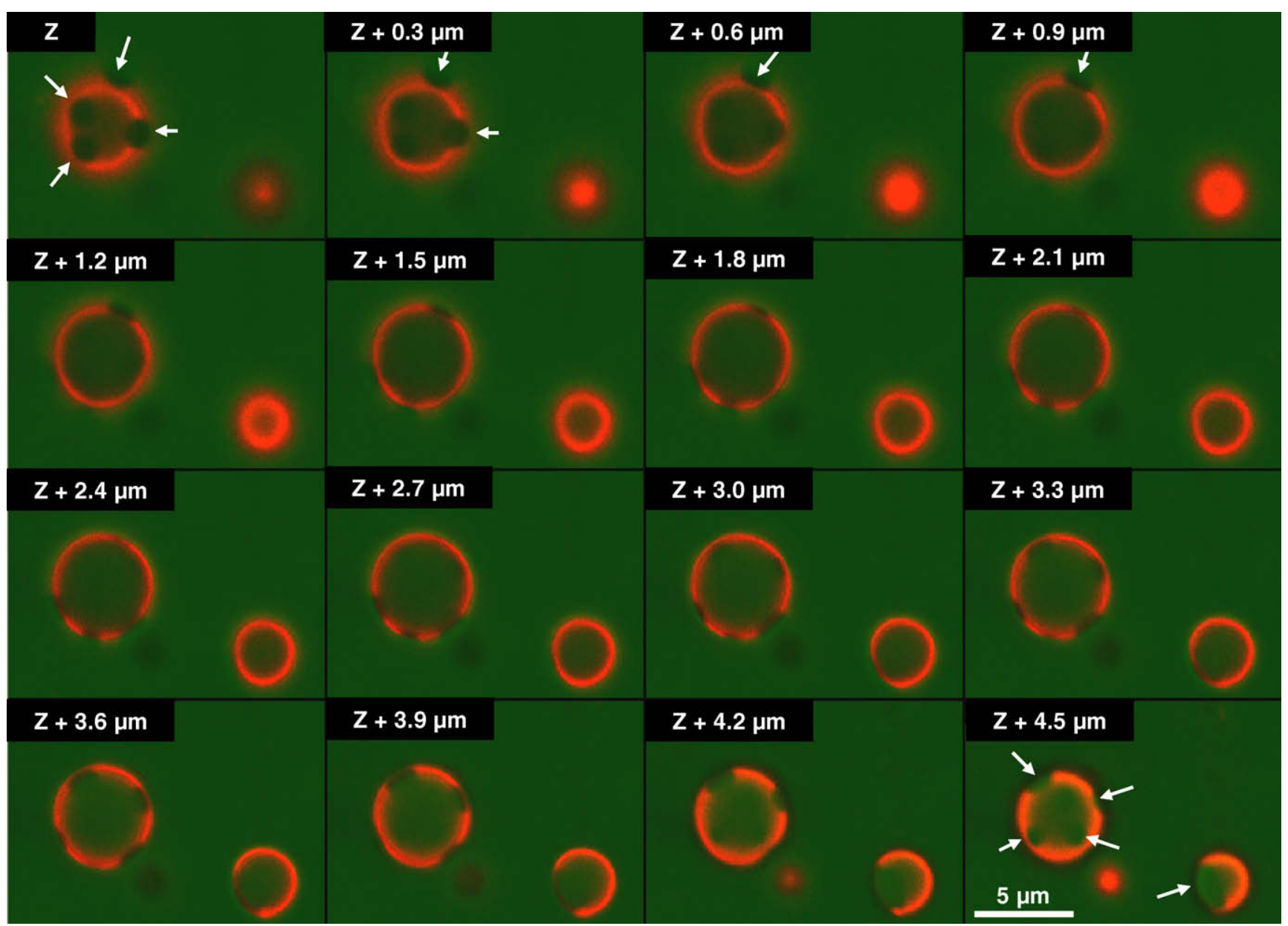

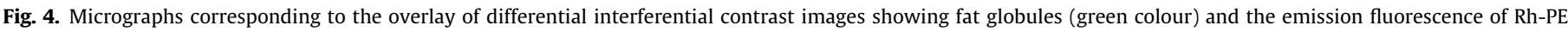

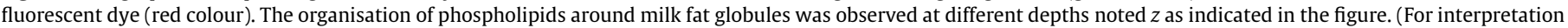
of the references to colour in this figure legend, the reader is referred to the web version of this article.)

Fig. 5 shows the changes in the organisation of the MFGM, which have been observed as a function of time at room temperature $\left(19 \pm 1{ }^{\circ} \mathrm{C}\right)$. At the microscopic level of CLSM, a lateral mobility of the MFGM constituents was characterised around fat globules. The non-fluorescent circular area dispersed in the MFGM moved as a function of time, as pointed by arrows in Fig. 5. However, the size and the shape of these micro-domains did not change as a function of time. CLSM experiments revealed that the MFGM is not a static biophysical system but corresponds to a highly dynamic organisation.

\subsection{Simultaneous localisation of polar lipids, glycolipids and glycoproteins in the MFGM}

CLSM with fluorescently labelled lectins, e.g. sugar-binding proteins, were used to investigate the localisation of the MFGM glycoproteins and glycolipids, in situ around fat globules. More precisely, the carbohydrate moieties located in the MFGM were labelled using a molecule with fluorescent properties (Alexa fluor 488) which is conjugated to concanavalin A (ConA) or to wheat germ agglutinin (WGA).

Fig. 6 shows the emission fluorescence of ConA detected by the confocal microscope (Fig. 6A), fat globules observed by DIC (Fig. 6B), and the overlay of the two images (Fig. 6C). ConA accumulated around the spherical fat globules, showing that the MFGM contains ConA-receptors disposed externally on the surface. As fat globules had various sizes they were not observed in the same $z$ depth of their volume. This is the reason why ConA was observed at the top or at the periphery of fat globules when they are observed in their equatorial plane. In this latter case, the core of fat globules corresponds to triacylglycerols (as already shown in Fig. 1). Fig. $6 B$ and $C$ shows that the surface of fat globules is not smooth, some holes in which ConA was present have been characterised. The spatial distribution of the oligosaccharides was heterogeneous around fat globules; the molecules may be organised in the form of a network in the external envelope of fat globules and as some patches revealed by the high emission fluorescence of ConA.

The hypothesis of the localisation of glycoproteins in the areas which are not labelled by Rh-PE (as observed in Figs. 2-5), was checked by developing the simultaneous labelling of the polar lipids and the oligosaccharides in the MFGM surrounding fat globules, using ConA (Fig. 7) and WGA (Fig. 8). Figs. 7 and 8 reveal that some areas of the MFGM contain glycoproteins organised as patches or as a network and protrude in the aqueous phase surrounding fat globules. These glycoproteins correspond to the glycocalyx surrounding fat globules. Moreover, Figs. 7 and 8 show that some areas of the MFGM are neither composed by polar lipids, glycolipids nor glycoproteins. The black circular areas have been observed (i) with the ConA and the Rh-PE fluorescent dyes (fat globule in the centre of the figure, Fig. 7) and (ii) with the WGA and the Rh-PE 

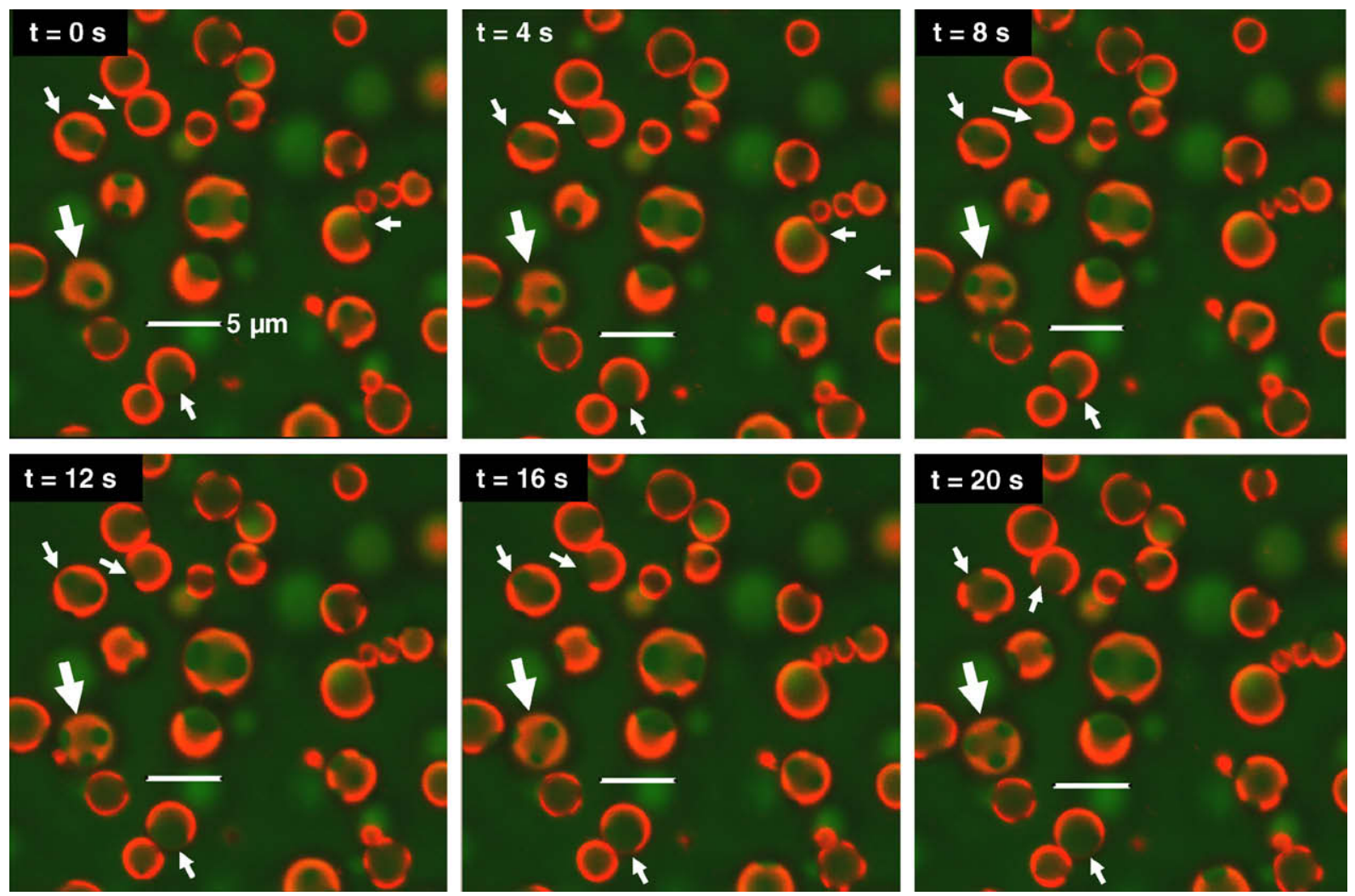

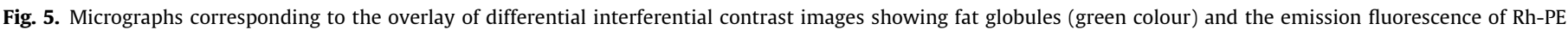

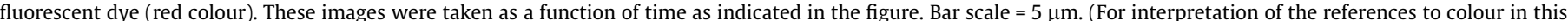
figure legend, the reader is referred to the web version of this article.)
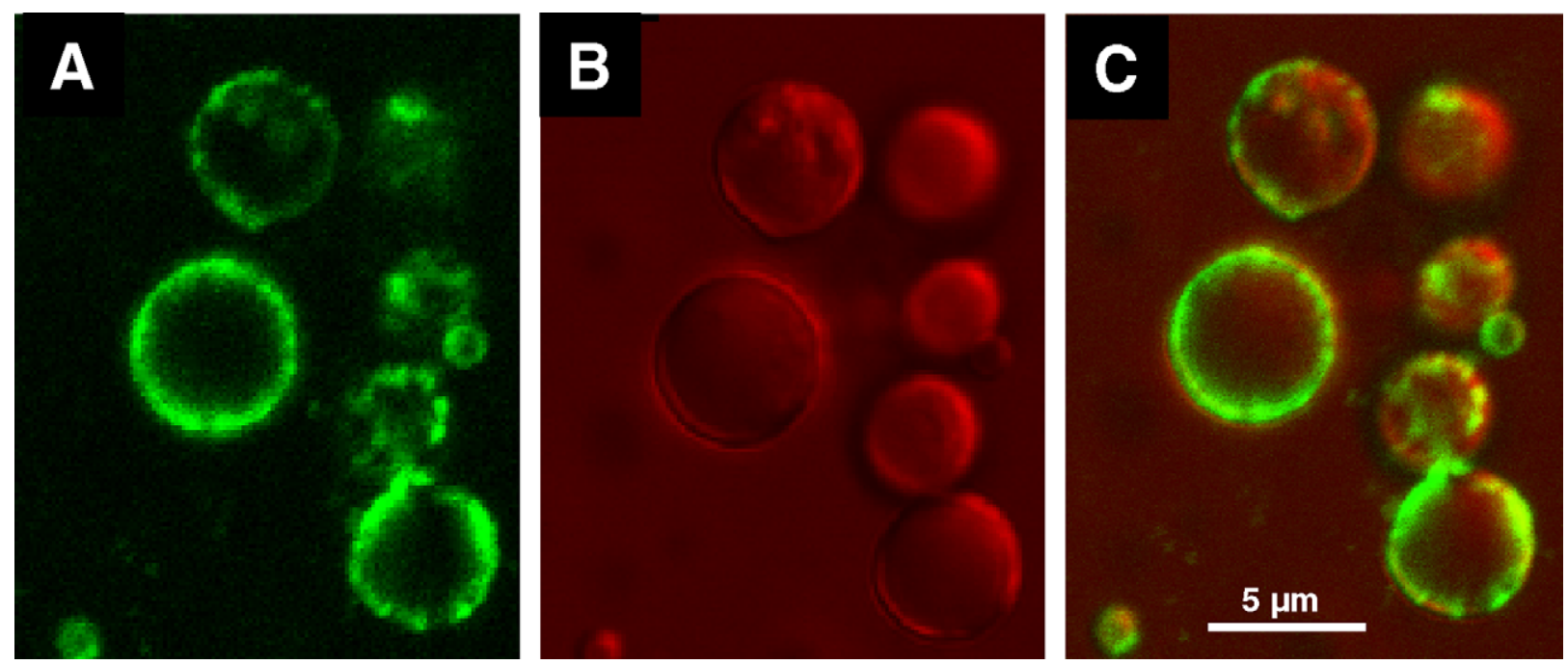

Fig. 6. Confocal laser scanning micrographs corresponding to (A) the emission fluorescence of concanavalin A alexa fluor 488, the fluorescent dye used to label the glycosylated molecules, (B) the differential interferential contrast image showing milk fat globules, and (C) overlay of the images A and B permitting the localisation of glycosylated molecules in the milk fat globule membrane surrounding fat globules.

fluorescent dyes (Fig. 8). These areas that remain black in the confocal micrographs may correspond to the localisation of sphingolipids in the MFGM, as discussed above. As carbohydrate moieties are present in glycoproteins and glycolipids, we can deduce from these CLSM investigations that the glycoproteins and the glycolipids accumulate in some areas and thus that they are not homogeneously distributed in the MFGM.

\section{Discussion}

For the last years, the MFGM has attracted the attention of scientists and industry because of its interesting functional and nutritional properties leading to potential health benefits. In this respect, increasing the knowledge about the lateral organisation of phospholipids, glycolipids and glycoproteins in the MFGM is of 

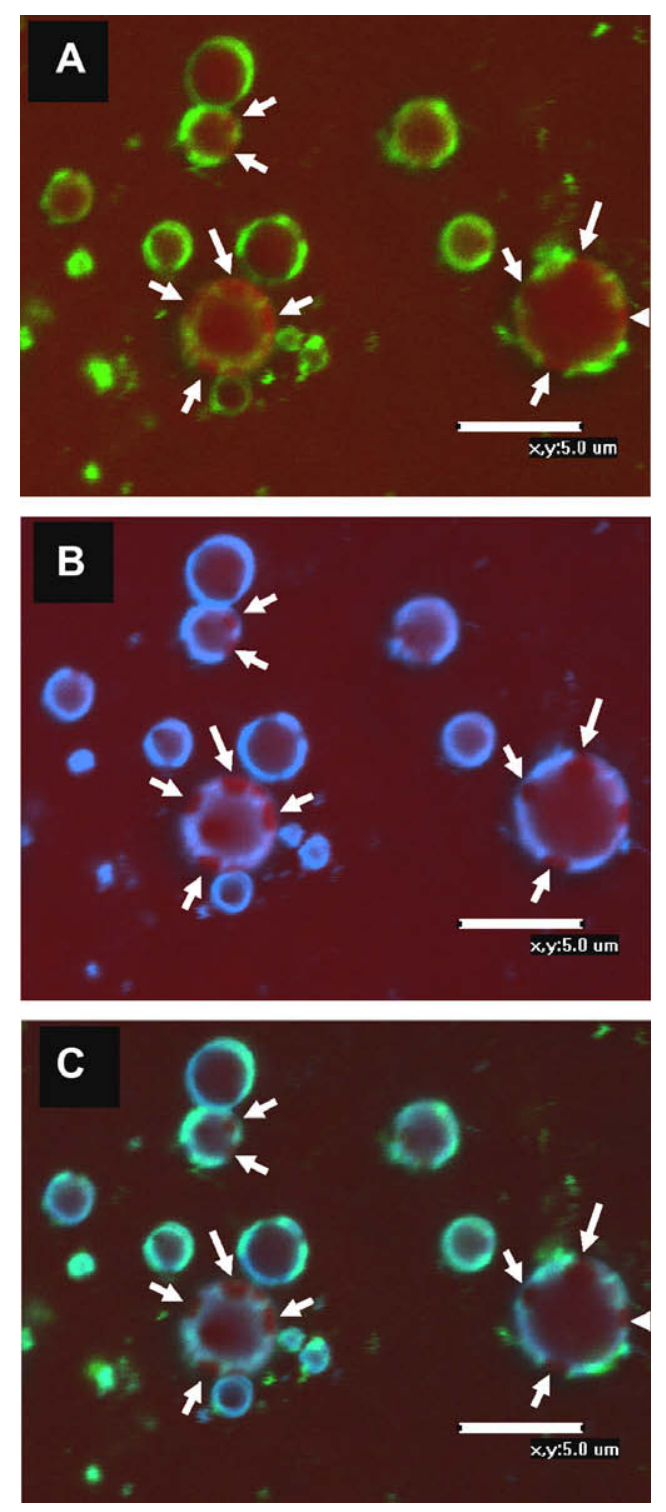

Fig. 7. Confocal laser scanning micrographs corresponding to $(A)$ the emission fluorescence of concanavalin A alexa fluor 488, the fluorescent dye used to label the glycosylated molecules, (B) the emission fluorescence of Rh-PE, the fluorescent dye used to label the polar lipids, and (C) the overlay of the images A and B with the differential interferential contrast image.

primary importance to enhance the understanding of their role in dairy products and in the gastro-intestinal tract.

\subsection{In situ characterisation of the size distribution, shape and composition of milk fat globules using optical and fluorescence microscopy techniques}

Optical microscopy with DIC allowed the characterisation of the size distribution and shape of fat globules dispersed in milk (Fig. 1). Both the size distribution and spherical shape of fat globules showed they were not altered nor deformed during the experiments. The investigations performed on milk fat globules using CLSM and pertinent fluorescent dyes allowed the localisation of triacylglycerols (Fig. 1), polar lipids (Figs. 2-5) and carbohydratecontaining molecules such as glycoproteins and glycolipids (Figs. 6-8).

From a methodological point of view, CLSM has advantages over conventional light microscopy among which are the possibility (i) to localise simultaneously glycoproteins, glycolipids and phospholipids in the MFGM, (ii) to perform observations as a function of time leading to the characterisation of dynamic processes (Fig. 5) and (iii) to perform in $z$-depth optical sectioning allowing the disturbance-free observation of the 3D structure of fat globules (Fig. 4). Moreover, the use of agarose increased the viscosity of the continuous phase and then limited the Brownian diffusion of fat globules dispersed in milk, which contributed to improve the quality of their characterisation using both DIC and CLSM techniques.

Nile Red fluorescent dye and both CLSM and DIC allowed the visualisation of the internal core of fat globules which is composed of triacylglycerols (Fig. 1). Our experiments support that Nile Red is a suitable dye for the localisation of hydrophobic lipids such as triacylglycerols by CLSM, as previously reported (Hassan, Frank, \& Corredig, 2002; Lopez, Briard-bion, et al., 2008; Lopez et al., 2007; Wendin, Langton, Caous, \& Hall, 2000).

The lectins ConA and WGA allowed the labelling of the MFGM glycolipids such as gangliosides and glycoproteins (Figs. 6-8). ConA selectively binds to alpha-mannopyranosyl and alpha-glycopyranosyl residues in glycoproteins. WGA selectively binds to Nacetylglucosamine and $\mathrm{N}$-acetylneuraminic acid (sialic acid) residues. According to the carbohydrate composition of the MFGM glycoproteins, we deduced that the lectins selectively bound at least to butyrophilin (20-40\% of the total MFGM protein; $2 \mathrm{~N}$-linked glycans), CD36 (N-linked glycans), MUC1 (O-linked glycans), MUC15 (N-linked glycans), and PAS6/7 (N- and O-linked glycans) which are the main glycoproteins of the MFGM (Keenan \& Mather, 2006). Also, WGA is able to bind to acidic glycosphingolipids (called gangliosides) which contain sialic acid. Using WGA, Evers et al. (2008) previously reported chemical and then structural heterogeneities in the MFGM.

The use of an exogenous phospholipid labelled with a fluorescent dye (Rh-PE) permitted the localisation of polar lipids in the membrane surrounding fat globules and to reveal heterogeneities in the MFGM that will be discussed below (Figs. 2-5). The size of the fluorescent moiety (rhodamine) of the exogenous phospholipid is more important than the size of the nitroxide group of the natural phospholipids (Fig. 9A). Thus, quantitative information from the fluorescent signal cannot be related to the thickness of the MFGM since it is overestimated. As special care is needed for the selection of lipid probes, we chose a probe that resembles its natural counterpart, i.e. milk phospholipids, without dye attached in the chain region to avoid changes in the hydrophilic/hydrophobic balance of the lipid molecule that would induce perturbation and change its ability to integrate into layers of membrane phospholipids. Head group labelled lipids, such as Rh-PE, allow the localisation of phospholipid layers, the hydrophobic tails of the lipid being firmly anchored with the membrane in an unperturbed manner (Maier, Oberle, \& Hoekstra, 2002). Phosphatidylethanolamine (PE) has often been employed as an anchor lipid for fluorescent probes such as dansyl, nitrobenzoxadiazole and rhodamine, allowing their covalent attachment to the amine group. Moreover, we used di-oleoyl-phosphatidylethanolamine (DOPE), which is the second most important phospholipid in milk, esterified with the major fatty acid found in milk PE, i.e. oleic acid (46.7\%; Keenan \& Patton, 1995). The polar groups of rhodamine, mainly $\mathrm{SO}_{3}^{-}$, increase the polarity of the PE, and then may facilitate its diffusion in milk to integrate the layers of phospholipids in the MFGM surrounding milk fat globules. The labelling of the MFGM was previously developed using Rh-PE to locate the phospholipids in Emmental cheese as reported in Lopez, Briard-Bion, et al. (2008). Evers et al. (2008) used DiLC ${ }_{18}(3)$-DS and FM4-64 as lipophilic probes to label the MFGM. Although these molecules are not phospholipids, Evers et al. (2008) interpreted that they were located in the outer layer of the MFGM bilayer of phospholipids. 

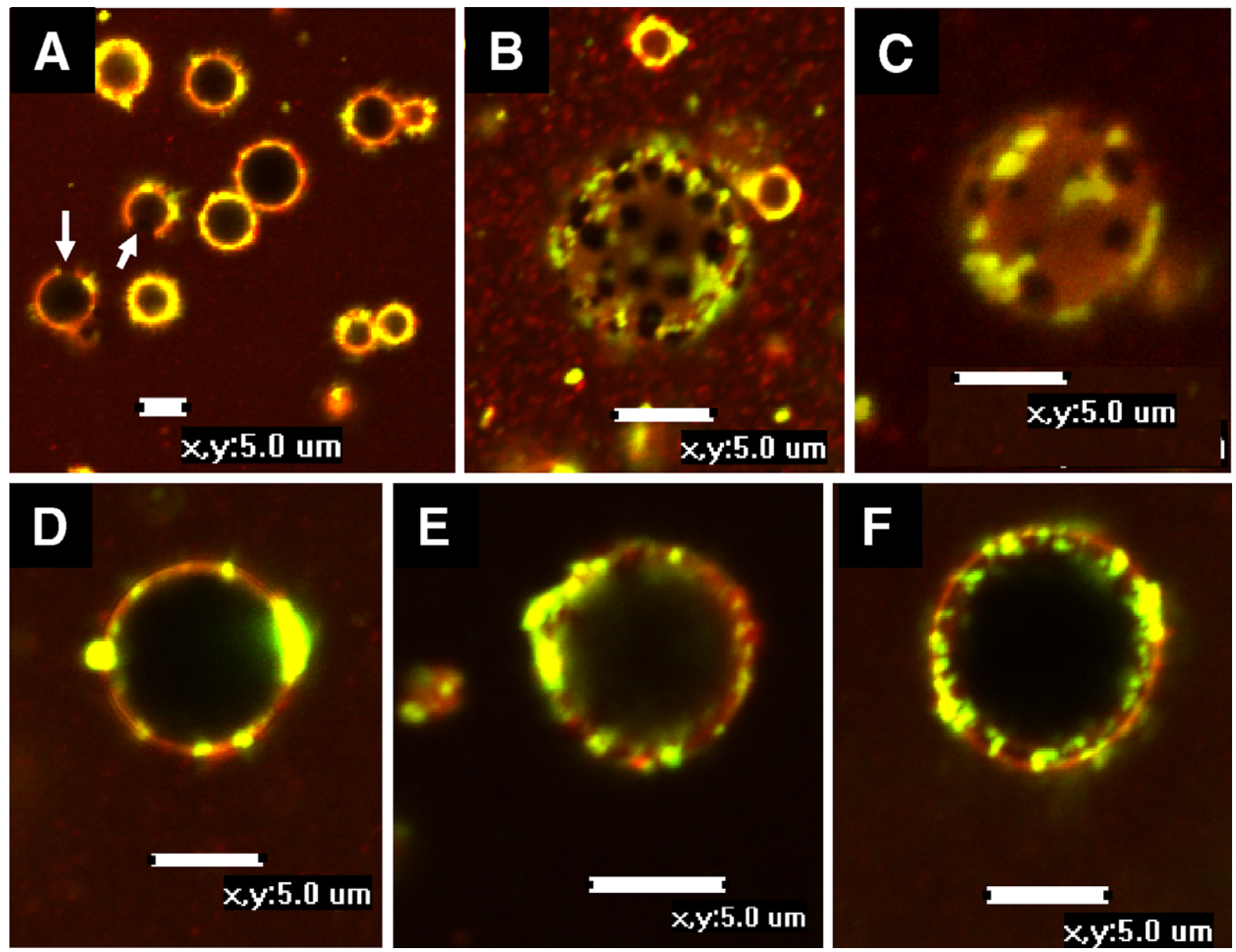

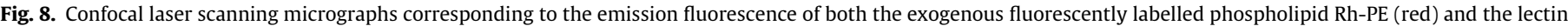

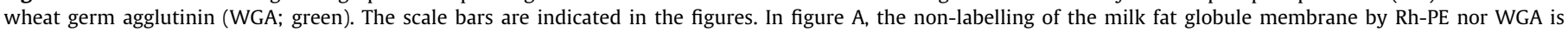
indicated by arrows. (For interpretation of the references to colour in this figure legend, the reader is referred to the web version of this article.)

\subsection{Heterogeneities in the lateral organisation of polar lipids and glycoproteins: presence of lipid rafts in the MFGM}

CLSM experiments performed in this study revealed that the fluorescent dyes Rh-PE, ConA and WGA were distributed heterogeneously around fat globules, which has been interpreted as heterogeneities in the structure and composition of the MFGM, as discussed below. Such heterogeneities in the MFGM have been recently reported by Evers et al. (2008) using different fluorescent dyes.

Figs. 2-5 show the absence of the exogenous fluorescent phospholipid Rh-PE in some areas of the MFGM. These areas may correspond: (i) to an absence of the MFGM bilayer of phospholipids around fat globules as previously reported by Evers et al. (2008), or (ii) to areas in which Rh-PE fluorescent dye was not allowed to insert the membrane. Considering the first hypothesis, it appears impossible that hydrophobic molecules such as triacylglycerols located in the core of fat globules could be directly in contact with the aqueous phase surrounding fat globules (Fig. 1). These areas may be constituted by lipid molecules which are not glycerophospholipids, such as (i) sphingolipids, or (ii) (glyco)proteins, or (iii) both. Evers et al. (2008) discussed the presence of glycosylated compounds from the inner layer of the MFGM. The hypothesis of the presence of areas enriched in proteins in the MFGM was checked by the labelling of glycoproteins which are the major proteins of the MFGM. Figs. 7 and 8 show that the micro-domains were devoided of phospholipids, glycolipids and glycoproteins.
Thus, the micro-domains characterised in the MFGM may correspond to rigid sphingolipid-enriched domains as discussed below.

Sphingolipids are characterised by a sphingoid base (sphingosine) in which the amino group is linked with a fatty acid and which is esterified with the phosphorylcholine headgroup. Moreover, sphingolipids (mainly sphingomyelin, SM) and glycosphingolipids (cerebrosides and gangliosides) differ from the glycerophospholipids in containing saturated long $\mathrm{N}$-acyl chains (C16:0, C22:0, C23:0, C24:0; Bitman \& Wood, 1990; Fong et al., 2007). As a result, sphingolipids have a much higher melting temperature $\left(35^{\circ} \mathrm{C}\right.$ according to Malmsten, Bergenstahl, Nyberg, \& Odham, 1994) than membrane glycerophospholipids which are rich in kinked unsaturated acyl chains (C18:1, C18:2, C18:3), essential for the fluidity of the MFGM (Bitman \& Wood, 1990; Fong et al., 2007). This composition of sphingolipids is responsible for a tight intermolecular interaction which allows them to readily pack together. Fluorescence microscopy revealed the formation of micro-domains which were entrapped into the bright continuum corresponding to the emission fluorescence of Rh-PE (Figs. 2-5). We interpreted these micro-domains as the phase separation and lateral segregation of sphingolipid-rich domains from glycerophospholipids due to their differential packing ability. This interpretation is not in agreement with the work reported by Evers et al. (2008) in which the absence of emission fluorescence observed by using lipohilic molecules was interpreted as an absence of the bilayer membrane. However, this interpretation is consistent with Goni and Alonso (2009) who reported that in mixtures with glyc- 


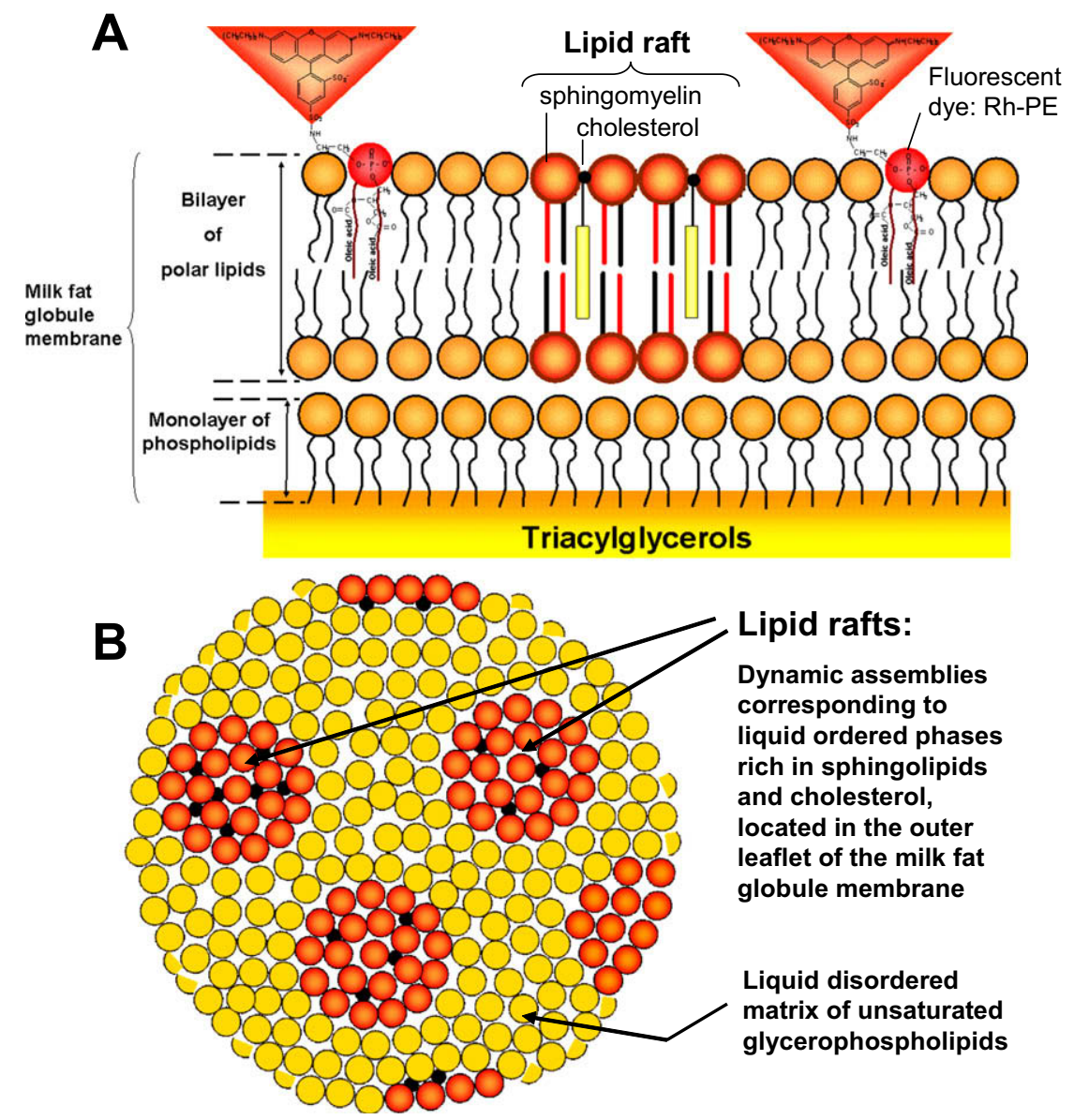

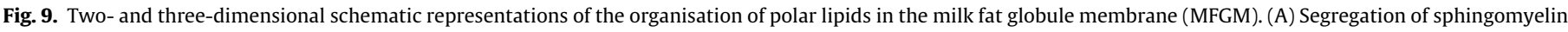

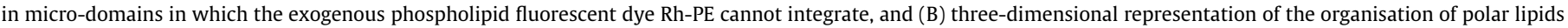
in the MFGM, showing the circular shape of the lipid rafts enriched in sphingomyelin. Not to scale.

erophospholipids, sphingolipids have two main effects, (i) they increase the molecular order of glycerophospholipids, and (ii) they give rise to lateral phase separation and domain formation. In fact, the fluorescent probe, Rh-PE, may have been excluded from the rigid sphingolipid-rich micro-domains (liquid-ordered phase). The membrane surrounding the rigid domains may consist mostly of glycerophospholipids (PE, PC, PI, PS) and is more fluid since RhPE integrated into the bilayer. A schematic representation of the lateral organisation of polar lipids in the MFGM based on our experimental observations is proposed in Fig. 9. Huang and Feigenson (1999) reported that lipids with large head groups such as SM have the capacity to accommodate small head-group lipids, such as cholesterol, in the ordered domain of the membrane. Thus, the cholesterol that is present in the MFGM $(0.46 \%$ of milk lipids; Bitman \& Wood, 1990) may be located in the SM-rich domains and play a role in the formation of the micro-domains (Fig. 9).

The morphology of the micro-domains, which have been characterised at room temperature $\left(19 \pm 1^{\circ} \mathrm{C}\right)$, corresponds to a circular shape. Domain shape is the result of a balance between line tension and dipole-dipole repulsion. In the MFGM, the former may dominate since circular domains with minimum domain boundaries arise. Moreover, the circular shape showed that the sphingolipidrich micro-domains corresponded to liquid-ordered phases and not to a gel phase in which lipids are semi-crystallised and form angles (Brown \& London, 1998). Fig. 6 shows that the rigid domains diffuse as independent entities in the outer leaflet of the MFGM. This is consistent with Sankaram and Thompson (1990) who reported that the preponderance of saturated hydrocarbon chains in sphingolipids such as SM allows the molecules to be tightly packed in the liquid-ordered phase (rigid domain), but nevertheless remain mobile in the plane of the membrane.

The identification of the lipid phases formed in the biological membranes requires the use of X-ray scattering methods (Luzzati, 1968). The identification of the lipid phases formed by glycerophospholipids and sphingolipids in the MFGM surrounding fat globules in situ in milk seems difficult since: (i) the polar lipids correspond to $0.2-1 \%$ of milk lipids located around the core of triacylglycerol molecules (98\% of milk lipids) and (ii) wide-angle X-ray signal shows the X-ray scattering of triacylglycerols in their liquid state, or the lamellar structure formed by triacylglycerols in their solid state (Lopez et al., 2002). The study of the lipid phases formed by milk polar lipids requires (i) the removing of the MFGM, which may induce artefacts or (ii) the study of models, e.g. construction of phase diagram with mixtures of purified milk polar lipids and cholesterol (Waninge, Nylander, Paulsson, \& Bergenstahl, 2003).

These discussions about the composition and organisation of the micro-domains observed in the MFGM using CLSM raise the question of the concept of the lipid raft. The concept of "raft" refers to a particular kind of micro-domain. A currently accepted definition of rafts is: small (10-20 nm), heterogeneous, highly dynamic, sterol- and sphingolipid-enriched domains that compartimentalise cellular processes (Pike, 2006). It is also well-accepted that tight acyl chain packing such as those formed by sphingolipids is a key feature of lipid raft organisation (Brown \& London, 1998). Although the size of the micro-domains that we observed using CLSM (Figs. $2-5$ ) is larger than those defined for cellular membranes, they may 


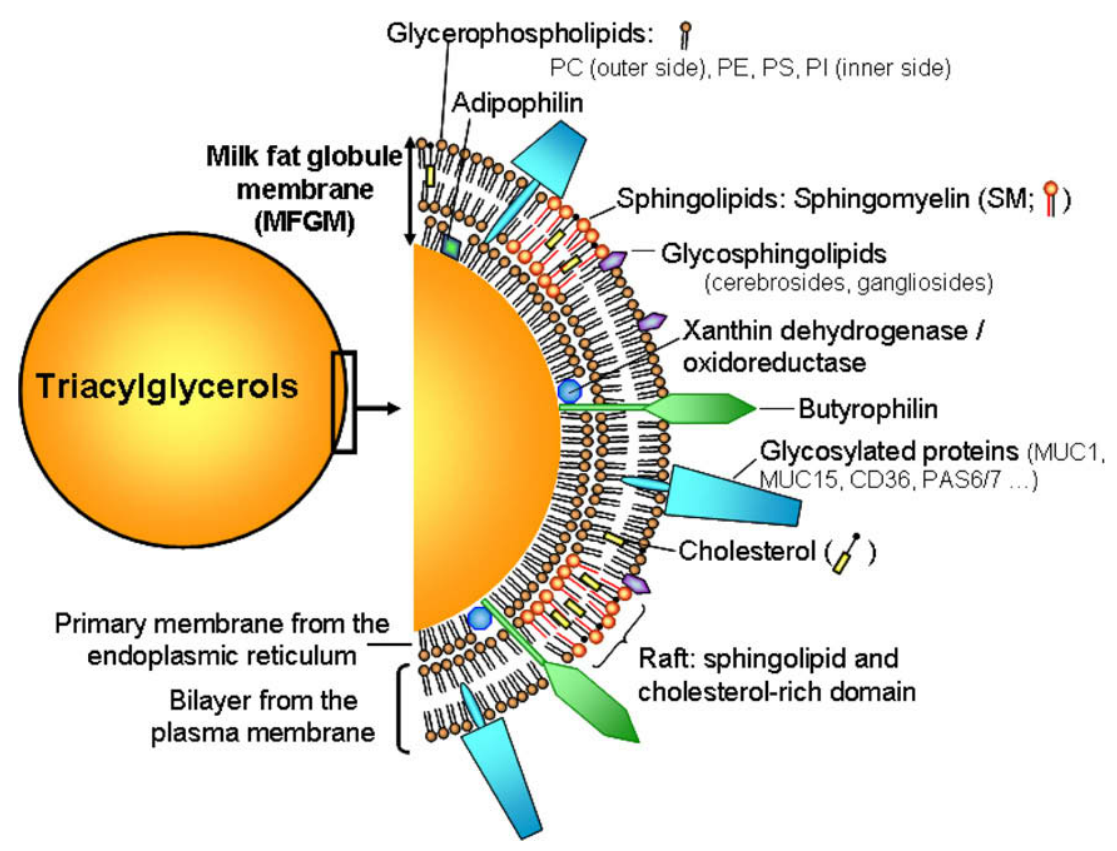

Fig. 10. Schematic representation of the structure of the milk fat globule membrane. Not to scale.

correspond to the existence of lipid rafts in the MFGM, consisting of dynamic assemblies of sphingolipids (mainly SM) and cholesterol in the outer leaflet of the bilayer (Figs. 9 and 10). The size of the lipid rafts observed in the MFGM was large enough to be resolved using CLSM (Figs. 2-5). Lipid rafts form distinct liquid-ordered phases in the MFGM, with a circular shape at room temperature, dispersed in a liquid-disordered matrix of unsaturated glycerophospholipids (Fig. 9). Simons and Ikonen (1997) reported that in polarised epithelial cells, lipid rafts accumulate in the apical plasma membrane. Since milk fat globules are secreted by the epithelial cells of the mammary gland, after being enveloped by the apical plasma membrane, it is not surprising to observe lipid rafts on the outer leaflet of the MFGM. The concept of lipid raft in the MFGM was previously proposed in Lopez, BriardBion, et al. (2008), from the observation of heterogeneities in the organisation of phospholipids around fat.

Since the molecules integrated in the lipid rafts are mainly sphingolipids, we can roughly estimate the fraction of the MFGM covered by lipid rafts through the relative proportion of sphingolipids (mainly SM) present in milk. Using laser light scattering, we calculated that the external surface of the MFGM corresponds to $3.8 \pm 0.1 \mathrm{~m}^{2} / \mathrm{g}$ fat and about $160 \mathrm{~m}^{2} / \mathrm{L}$ milk. Considering the three layers of polar lipids, the surface is about $11.4 \mathrm{~m}^{2} / \mathrm{g}$ fat and about $480 \mathrm{~m}^{2} / \mathrm{L}$ milk. If we consider that SM (i) corresponds to $25 \%$ of polar lipids (Christie, 1995; Lopez, Briard-Bion, et al., 2008), (ii) is only located in the outer leaflet of the MFGM, then it corresponds to $75 \%$ of the surface of fat globules $\left(2.85 \mathrm{~m}^{2} / \mathrm{g}\right.$ fat and $120 \mathrm{~m}^{2} / \mathrm{L}$ milk). If we consider that SM is located in the two leaflets of the bilayer of phospholipids, then it corresponds to $50 \%$ of the external surface of fat globules. This is consistent with the observations performed using CLSM (Figs. 3-5 and 8). The glycerophospholipids (PE, PC, PI, PS) are present in the three layers of the MFGM and located around the lipid rafts in the outer leaflet (Fig. 9).

Regarding the carbohydrate-containing molecules, we characterised the spatial distribution of ConA and WGA receptor sites in the MFGM, in situ in milk. According to their chemical composition and previous discussion, the glycosphingolipids may be located within lipid rafts dispersed in the MFGM. However, CLSM observations performed using both Rh-PE and lectins did not reveal the localisation of glycosphingolipids within the lipid rafts.
The hypotheses are: (i) that glycosphingolipids are located at the periphery of lipid rafts, between SM and the glycerophospholipids (Figs. 7 and 8) and (ii) that glycosphingolipid are quantitatively minor low molecular weight components of the MFGM and that their presence was not detected (even when sialic acid labelled using WGA). CLSM experiments also revealed that the glycoproteins are not localised within the lipid rafts. Lipid rafts can include or exclude proteins to variable extent depending on their composition, structure and then affinity for the raft. Also, proteins may have a short residency time in the rafts, spending most of their time outside the rafts. In our experiments, glycoproteins did not appear to be integrated in the lipid rafts (Figs. 7 and 8). This is in agreement with Evers et al. (2008) who observed circular domains which were not fluorescent when they used WGA-488 to label the glycolipids and glycoproteins in the ovine MFGM. However, Evers et al. (2008) did not interpret the circular domains as lipid rafts but as sections of the MFGM in which the bilayer was absent. CLSM experiments showed that the glycoproteins are not distributed homogeneously over the external membrane surface (Figs. 6-8). As butyrophilin is by far the main glycosylated protein of the MFGM (Mather, 2000), the emission fluorescence of the lectins around fat globules may correspond mainly to this transmembrane protein that is glycosylated in the part of the molecule exposed on the external surface of the membrane. Our results support the work of Robenek et al. (2006) who observed the organisation of butyrophilin in a punctuated ring-like pattern at the surface of fat globules using immunofluorescence microscopy. CLSM experiments allowed the visualisation of the glycocalyx surrounding bovine milk fat globules, since the carbohydrate residues of glycolipids and glycoproteins are located on the external surface of the MFGM (Fig. 7).

The structure of the MFGM bilayer results from the mechanisms of secretion of fat globules. However, as the apical plasma membrane of the mammary gland secretory cell is a highly dynamic membrane, the fat globule secretion process is expected to involve many biochemical and physiological changes (Evers, 2004). Hence, significant rotational and lateral diffusion, and possibly trans-layer movement (flip-flop) of membrane compounds are possible. All these changes suggest a highly dynamic process at the molecular level which may affect the lateral organisation of the MFGM. Also, 
during secretion of fat globules, changes in the curvature of the bilayer membrane occur, the degree of which will depend on the size of fat globules. However, we did not observe changes in the lateral organisation of the main components of the MFGM whatever the size of fat globules (from 0.5 to $10 \mu \mathrm{m}$ ).

On the basis of our experimental results obtained using CLSM and pertinent fluorescent dyes, we propose a new model for the organisation of the MFGM (Fig. 10). This model integrates the presence of the rigid lipid rafts in the MFGM, which are surrounded by the more fluid part of the MFGM composed of the glycerophospholipids and the glycoproteins.

\subsection{Heterogeneous distribution of polar lipids and glycoproteins in the} MFGM: new insights to understand their physiological role

The MFGM is undoubtedly a source of bioactive molecules such as glycerophospholipids, gangliosides, cerebrosides, cholesterol and glycoproteins. As a result of the present study and for future research focus, we do think that the relevant question is: what is the role played by the lateral organisation of MFGM components around milk fat globules on their physiological functions in the gastro-intestinal tract?

The glycoproteins of the MFGM play important biological roles, as reviewed in Spitsberg (2005). There is some evidence that MUC1 plays an immuno-protective role in the suckling neonate by sequestering pathogenic microorganisms (for review, see Mather, 2000). CD36 is known to have a number of diverse functions in the vascular and hemopoietic systems, mostly as receptor (Mather, 2000). The human homologue of PAS6/7 binds to rotaviruses and may protect the gastro-intestinal tract of the suckling young from infection (Peterson, Scallan, Ceriani, \& Hamosh, 2001). The location of these proteins in the glycocalyx surrounding fat globules may contribute to elucidate the physiological role played by the MFGM, which is altered after homogenisation of milk.

Lipid rafts have been detected in living cells and for the first time in the outer leaflet of the MFGM, which originates from the mammary epithelial cells. The role of lipid rafts in the outer leaflet of the MFGM is an important outstanding question. Lipid rafts are suggested to play important roles in a number of cellular processes as diverse as signal transduction, membrane traffic and pathogen entry (Simons \& Ikonen, 1997). Rafts can serve as docking sites for certain pathogens and toxins (Fivaz, Abrami, \& van der Goot, 1999). The protective effect of sphingolipids against bacterial toxins and infection has also been demonstrated (Rueda, Sabatel, \& Maldonado, 1998; Sprong, Hulstein, \& Van der Meer, 2002). Recently, Noh and Koo (2004) have demonstrated that sphingomyelin is an effective inhibitor of intestinal absorption of cholesterol in rats. The inhibition has been explained by a direct inhibiting effect of the highly saturated long chains of fatty acyl groups of milk sphingomyelin on the rate of luminal lipolysis, micellar solubilisation and transfer of micellar lipids to the enterocyte. The ability of sphingomyelin molecules to organise in liquid-ordered domains such as lipid rafts which has been revealed in this study could explain the physiological role of milk sphingolipids in the gastrointestinal tract. Also the glycerophospholipids which self-organise as a liquid-disordered matrix in the MFGM could participate in the micellar solubilisation and absorption of hydrophobic and amphiphilic molecules (antioxidant molecules, lipopolysaccharides...). From this point of view, the MFGM could be considered as a potential delivery system of liposoluble nutrients in the intestine. The glycerophospholipids and sphingolipids may serve very different biological functions, determined by their different impact on the MFGM physical properties. The rigid sphingolipid-rich micro-domains may confer a higher fragility compared to the fluid glycerophospholipid-rich domains of the MFGM. This fragility may correspond to the break down point of the MFGM during process- ing of milk (for example during churning). The lateral organisation of polar lipids and the presence of lipid rafts in the MFGM may also affect the accessibility of triacylglycerols to the lipolytic enzymes, particularly the gastric and pancreatic lipases during digestion. Goni and Alonso (2009) showed that the enzymes prefer to adsorb on the fluid lipid disordered domains which are rich in glycerophospholipids. The increased knowledge of the lateral organisation of polar lipids, glycolipids and glycoproteins in the MFGM will contribute to a better understanding of their role during digestion of milk fat globules.

\section{Conclusion}

This study provides new information regarding the lateral organisation of polar lipids, glycolipids and glycoproteins in the MFGM. Using the coupling of DIC and CLSM with pertinent fluorescent molecular probes, e.g. the exogeneous phospholipid Rh-PE and the lectins ConA and WGA, the experiments revealed chemical and structural heterogeneities in the MFGM in its native environment, e.g. around fat globules in milk. The presence of rigid lipid rafts rich in sphingolipids and characterised by a circular shape at room temperature was evidenced in the MFGM. The glycerophospholipids and the glycoproteins are located around the lipid rafts, in the more fluid part of the MFGM. The MFGM may represent a model system for studying structure and dynamics of biomembranes.

\section{Acknowledgements}

Benoit ROBERT (INRA UMR STLO, Rennes, France) is acknowledged for providing milk samples. Florence ROUSSEAU and Olivia MENARD (INRA UMR STLO, Rennes, France) are acknowledged for zeta potential experiments, fat globule size measurements and fat content determinations in the experimental milks. Dr. Christelle CEBO (INRA, Jouy en Josas, France) is acknowledged for interesting discussions about MFGM glycoproteins.

\section{References}

Bitman, J., \& Wood, D. L. (1990). Changes in milk phospholipids during lactation. Journal of Dairy Science, 73, 1208-1216.

Brown, D. A., \& London, E. (1998). Functions of lipid rafts in biological membranes. Annual Review of Cell and Developmental Biology, 14, 111-136.

Christie, W. W. (1995). Composition and structure of milk lipids. In P. F. Fox (Ed.) Advanced dairy chemistry. Lipids (Vol. 2, pp. 1-37). New York: Chapman \& Hall.

Danthine, S., Blecker, C., Paquot, M. Innocente, N., \& Deroanne, C. (2000). Evolution des connaissances sur la membrane du globule gras du lait: Synthèse bibliographique. Lait, 80, 209-222.

Dewettinck, K., Rombaut, R., Thienpont, N., Le, T. T., Messens, K., \& van Camp, J. (2008). Nutritional and technological aspects of milk fat globule membrane material. International Dairy Journal, 18, 436-457.

Evers, J. M. (2004). The milkfat globule membrane - compositional and structural changes post secretion by the mammary secretory cell. International Dairy Journal, 14, 661-674.

Evers, J. M., Haverkamp, R. G., Holroyd, S. E., Jameson, G. B., Mackenzie, D. D. S., \& McCarthy, O. J. (2008). Heterogeneity of milk fat globule membrane structure and composition as observed using fluorescence microscopy techniques. International Dairy Journal, 18, 1081-1089.

Fivaz, M., Abrami, L., \& van der Goot, F. (1999). Landing on lipid rafts. Trends Cell Biology, 9, 212-213.

Fong, B. Y., Norris, C. S., \& MacGibbon, A. K. H. (2007). Protein and lipid composition of bovine milk-fat-globule membrane. International Dairy Journal, 17, 275-288.

Franke, W. W., Heid, H. W., Grund, C., Winter, S., Freudenstein, C., Schmid, E., et al (1981). Antibodies to the major insoluble milk fat globule membraneassociated protein: Specific location in apical regions of lactating epithelial cells. Journal of Cell Biology, 89, 485-494.

Goni, F. M., \& Alonso, A. (2009). Effect of ceramide and other simple sphingolipids on membrane lateral structure. Biochimica and Biophysica Acta, 1788, 169-177.

Hassan, A. N., Frank, J. F., \& Corredig, M. (2002). Microstructure of feta cheese made using different cultures as determined by confocal scanning laser microscopy. Journal of Food Science, 67, 2750-2753.

Heid, H. W., \& Keenan, T. W. (2005). Intracellular origin and secretion of milk fat globules. European Journal of Cell Biology, 84, 245-258. 
Horisberger, M., Rosset, J., \& Vonlanthen, M. (1977). Location of glycoproteins on milk fat globule membrane by scanning and transmission electron microscopy, using lectin-labelled gold granules. Experimental Cell Research, 109, 361-369.

Huang, J., \& Feigenson, G. W. (1999). A microscopic interaction model of maximum solubility of cholesterol in lipid bilayers. Biophysical Journal, 76, 2142-2157.

Keenan, T. W. \& Mather, I. H. (2006). Intracellular origin of milk fat globules and the nature of the milk fat globule membrane. In P. F. Fox, \& P. L. H. McSweeney (Eds.), Advanced dairy chemistry. Lipids (3rd ed.) (Vol. 2, pp. 137-171). New York, NY, USA: Springer.

Keenan, T. W. \& Patton, S. (1995). The milk fat globule membrane. In R. G. Jensen (Ed.), Handbook of milk composition (pp. 5-50). San Diego, CA: Academic Press.

Lopez, C., Bourgaux, C., Lesieur, P., Bernadou, S., Keller, G., \& Ollivon, M. (2002). Thermal and structural behavior of milk fat: 3. Influence of cream cooling rate and droplet size. Journal of Colloid and Interface Science, 254, 64-78.

Lopez, C., Briard-Bion, V., Beaucher, E., \& Ollivon, M. (2008). Multiscale characterization of the organization of triglycerides and phospholipids in Emmental cheese: From the microscopic to the molecular level. Journal of Agricultural and Food Chemistry, 56, 2406-2414.

Lopez, C., Briard-Bion, V., Ménard, O., Rousseau, F., Pradel, P., \& Besle, J.-M. (2008). The phospholipid, sphingolipid and fatty acid compositions of the milk fat globule membrane are modified by diet. Journal of Agricultural Food Chemistry. 56, 5226-5236.

Lopez, C., Camier, B., \& Gassi, J.-Y. (2007). Development of the milk fat microstructure during the manufacture and ripening of Emmental cheese observed by confocal laser scanning microscopy. International Dairy Journal, 17 235-247.

Luzzati, V. (1968). X-ray diffraction studies of water-liquid systems. In D. Chapman (Ed.), Biological membranes (Vol. 1, pp. 71-123). London: Academic Press.

Maier, O., Oberle, V., \& Hoekstra, D. (2002). Fluorescent lipid probes: Some properties and applications (a review). Chemistry and Physics of Lipids, 116, $3-18$.

Malmsten, M., Bergenstahl, B., Nyberg, L., \& Odham, G. (1994). Sphingomyelin from milk, characterisation of liquid crystalline liposome and emulsion properties. Journal of the American Oil Chemists' Society, 71(9), 1021-1026.

Mather, I. H. (2000). A review and proposed nomenclature for major milk proteins of the milk-fat globule membrane. Journal of Dairy Science, 83, 203-247.
Michalski, M.-C., Michel, F., Sainmont, D., \& Briard, V. (2001). Apparent $\xi$-potential as a tool to assess mechanical damages to the milk fat globule membrane. Colloids and Surfaces B: Biointerfaces, 23, 23-30.

Noh, S. K., \& Koo, S. I. (2004). Milk sphingomyelin is more effective than egg sphingomyelin in inhibiting intestinal absorption of cholesterol and fat in rats. Journal of Nutrition, 134, 2611-2616.

Peterson, J. A., Scallan, C. D., Ceriani, R. L., \& Hamosh, M. (2001). Structural and functional aspects of three major glycoproteins of the human milk fat globule membrane. In D. S. Newburg (Ed.), Bioactive components of human milk (pp. 179-192). New York: Kluwer Academic/Plenum Publishers.

Pike, L. J. (2006). Rafts defined: A report on the keystone symposium on lipid rafts and cell function. Journal of Lipid Research, 47, 1597-1598.

Reinhardt, T. A., \& Lippolis, J. D. (2006). Bovine milk fat globule membrane proteome. Journal of Dairy Research, 73, 406-416.

Robenek, H., Hofnagel, O., Buers, I., Lorkowski, S., Schnoor, M., Robenek, M. J., et al. (2006). Butyrophilin controls milk fat globule secretion. Proceedings of the National Academy Science USA, 103, 10385-10390.

Rombaut, R., Camp, J. V., \& Dewettinck, K. (2005). Analysis of phospho- and sphingolipids in dairy products by a new HPLC method. Journal of Dairy Science, $88,482-488$.

Rueda, R., Sabatel, J. L., \& Maldonado, J. (1998). Addition of gangliosides to an adapted milk formula modifies levels of fecal Escherichia coli in preterm newborn infants. Journal of Pediatric, 133, 90-94.

Sankaram, M. B., \& Thompson, B. E. (1990). Interaction of cholesterol with various glycerophospholipids and sphingomyelin. Biochemistry, 29, 10670-10675.

Simons, K., \& Ikonen, E. (1997). Functional rafts in cell membrane. Nature, 387. 569-572.

Singer, S. J., \& Nicolson, G. L. (1972). The fluid mosaic model of the structure of cell membranes. Science, 175, 720-731.

Spitsberg, V. J. (2005). Bovine milk fat globule membrane as a potential nutraceutical. Journal of Dairy Science, 88, 2289-2294.

Sprong, R. C., Hulstein, M. F. E., \& Van der Meer, R. (2002). Bovine milk fat composition inhibit food-borne pathogens. International Dairy Journal, 12, 209-215.

Waninge, R., Nylander, T., Paulsson, M., \& Bergenstahl, B. (2003). Phase equilibra of model milk membrane lipid systems. Chemistry and Physics of Lipids, 125, 59-68.

Wendin, K., Langton, M., Caous, L., \& Hall, G. (2000). Dynamic analysis of sensory and microstructure properties of cream cheese. Food Chemistry, 71, 363-378. 